\title{
THE STRUCTURE OF HYPERREDUCIBLE TRIANGULAR ALGEBRAS
}

\author{
JOHN R. SCHUE
}

Introduction. In [5] Kadison and Singer have defined triangular algebras of operators on a Hilbert space and have investigated a number of their properties with the major emphasis on classification and examples. It is the purpose of this paper to give a new construction for the hyperreducible algebras which gives some additional insight in to their structure. In particular, Theorem 3 shows that any algebra $J$ of this form with diagonal $a$ can be written $J=Q+S$, where $\delta$ is the weak closure of an increasing sequence of weakly closed nilpotent ideals.

1. Notation and spectral theory. $a$ will denote a fixed maximal abelian self-adjoint subalgebra of a factor $B$ acting on a separable Hilbert space F. For $X \in B$, define the operators $L_{X}, R_{X}, D_{X}$ on $B$ by the equations $L_{x} Y=X Y, R_{x} Y=Y X$, and $D_{x} Y=X Y-Y X$ for $Y \in ß$. If the uniform topology is used on $B$, each of these operators is bounded and $\left\|L_{x}\right\|=\left\|R_{x}\right\|=\|X\|$. Furthermore, $D_{x}$ is a derivation on $B$. Let $\&$ and $Q$ denote the sets of $L_{A}, R_{A}, A \in Q$, respectively. Then $\mathscr{L}$ and $\mathcal{R}$ are commutative Banach algebras and each is isomorphic to $a$ under the natural mappings. Let $\mathcal{C}$ be the uniformly closed algebra of operators on $B$ generated by $\mathcal{L} \cup$ R. Thus $\mathbb{e}$ contains the identity operator.

We let $\Delta$ denote the spectrum of $a$, i.e., the set of all homomorphisms of $a$ onto the complex numbers. $\Delta$ is a compact Hausdorff space under the Gel'fand topology and $Q, \mathcal{L}$, and $R$ all may be identified with the continuous complex-valued functions on $\Delta$. If we let $\Gamma$ denote the spectrum of $\mathfrak{e}$ then $\Gamma$ is also compact and Hausdorff in the Gel'fand topology.

TheOREM 1. Suppose $\gamma \in \Gamma$ and $\alpha, \beta$ are the restrictions of $\gamma$ to $\&$ and $\Re$, respectively. Then the mapping $\gamma \rightarrow \gamma^{\prime}=(\alpha, \beta)$ is a homeomorphism of $\Gamma$ onto the product space $\Delta \times \Delta$.

For the proof we need two lemmas.

Lemma 1. For $X, Y \in ৫, L_{x} R_{y}=0$ implies $X=0$ or $Y=0$.

Proof. Let $\mathscr{g}=\left\{z: L_{z} R_{y}=0\right\}$. Then $X \in \mathfrak{g}$ and $\mathscr{g}$ is a weakly closed

Received by the editors January 25, 1963 and, in revised form, May 27, 1963. 
two-sided ideal of $B$ so that either $g=\{0\}$ or $\mathscr{g}=B$. In the first case $X=0$ and in the second $Y=0$.

LEMMA 2. Let $\mathfrak{C}_{0}$ be the set of all finite linear combinations of the form $\sum \lambda_{i} L_{E_{i}} R_{F_{i}}$ where $E_{i}$ and $F_{i}$ are projections in a and either $E_{i} E_{j}=0$ or $F_{i} F_{j}=0$ for $i \neq j$. Then $\mathfrak{C}_{0}$ is a dense subalgebra of $\mathfrak{e}$.

Proof. The usual operations with projections show fairly directly that $\mathfrak{C}_{0}$ is a subalgebra. In fact, with the obvious modifications, the proof is like that showing that the set of all finite linear combinations of characteristic functions of sub-rectangles (with sides parallel to the axes) of a given planar rectangle forms an algebra. From the spectral theorem applied to $A$ and $B$ it is easy to see that each $L_{A} R_{B}(A, B \in Q)$ can be approximated uniformly by elements of $\mathfrak{C}_{0}$ and thus $\mathfrak{C}_{0}$ is dense in $\mathrm{e}$.

Proof of Theorem 1. For $\gamma \in \Gamma$ and an element of $\mathfrak{C}_{0}, \gamma\left(\sum L_{E_{i}} R_{F_{i}}\right)$ $=\sum \alpha\left(E_{i}\right) \beta\left(F_{i}\right)$. Since $\mathfrak{C}_{0}$ is uniformly dense in $\mathfrak{C}$ this means that $\gamma$ is determined uniquely by $\alpha$ and $\beta$ and thus the correspondence defined on $\Gamma$ is one-one. If $\left\{\gamma_{j}\right\}$ is a directed sequence in $\Gamma$ converging to $\gamma$ and $\gamma_{j}=\left(\alpha_{j}, \beta_{j}\right), \gamma^{\prime}=(\alpha, \beta)$ then $\gamma_{j}(A) \rightarrow \gamma(A)$ for all $A \in Q$ so that $\left\{\alpha_{j}\right\}$ and $\left\{\beta_{j}\right\}$ converge to $\alpha$ and $\beta$, respectively. Thus $\gamma_{j}^{\prime} \rightarrow \gamma^{\prime}$ and the mapping is continuous. Since $\Gamma$ is compact, the image is compact and it only remains to prove that this image is all of $\Delta \times \Delta$.

Thus suppose $(\alpha, \beta) \in \Delta \times \Delta$ and $C=\sum \lambda_{i} L_{E_{i}} R_{F_{i}} \in \mathcal{C}_{0}$. Let $\gamma(C)$ $=\sum \lambda_{i} \alpha\left(E_{i}\right) \beta\left(F_{i}\right)$. If $\gamma(C) \neq 0$ there is a $j$ with $\alpha\left(E_{j}\right)=\beta\left(F_{j}\right)=1$. For $i \neq j$ either $E_{i} E_{j}=0$ or $F_{i} F_{j}=0$ so that, in any case, $\alpha\left(E_{i}\right) \beta\left(F_{i}\right)=0$ and hence $\gamma(C)=\lambda_{j}$. Since $L_{E_{j}} R_{F_{j}} \neq 0$, there is an $X \in B$ with $\|X\|=1$ and $E_{j} X F_{j}=X$. Necessarily $E_{i} X F_{i}=0$ for $i \neq j$ and thus $C(X)=\lambda_{j} X$ so that $\|C\| \geqq\left|\lambda_{j}\right|=|\gamma(C)|$. The mapping $C \rightarrow \gamma(C)$ will be linear on $\mathfrak{C}_{0}$ and the argument above shows that it is well-defined and normdecreasing. $\gamma$ is clearly multiplicative on $\mathfrak{C}_{0}$ and thus can be extended uniquely to all of $\mathcal{e}$ giving a homomorphism of $\mathcal{C}$ also denoted by $\gamma$. Since $\gamma\left(L_{1}\right)=1, \gamma$ is nontrivial and thus belongs to $\Gamma$. Since $\gamma^{\prime}=(\alpha, \beta)$ the proof is complete.

\section{Construction of triangular algebras.}

Definitions. Because of the separability assumption there exists a self-adjoint element $A \in Q$ such that every element of $Q$ is a bounded measurable function of $A$. Thus $a=\left\{X: D_{A} X=0\right\}$. We choose a fixed $A$ with these properties and, without any significant loss of generality, assume that the spectrum of $A$ lies in the interval $[0,1]$ and contains both end points. For $\gamma=(\alpha, \beta) \in \Gamma$ let $f(\gamma)=\gamma\left(D_{A}\right)$ $=\alpha(A)-\beta(A)$. Thus, relative to $\mathrm{e}$, the spectrum of $D_{A}$ is given by 
the range of $f$, a subset of $[-1,1]$. Since the range of $f$ is real and compact, it does not separate the plane, and the general theory of Banach algebras implies that the spectrum of $D_{A}$, relative to the algebra of all bounded operators on $B$, is also given by the range of $f$.

For real $\lambda$ and $\epsilon>0$, let $S(\lambda, \epsilon)$ denote the linear manifold consisting of all $X \in \Theta$ for which there is a constant $K_{x}$ such that $\left\|\left(D_{A}-\lambda\right)^{n} X\right\|$ $\leqq \epsilon^{n} K_{x}$ for $n=1,2, \cdots$. Then $S(\lambda, \epsilon)$ is invariant under all bounded operators on $B$ which commute with $D_{A}$. For a compact subset $M$ of the real numbers, let $S(M, \epsilon)$ be the closure in the weak operator topology of $B$ of the manifold spanned by $\{S(\lambda, \epsilon): \lambda \in M\}$ and let $S(M)=\bigcap_{\epsilon>0} S(M, \epsilon)$. Finally, for any Borel set $N$ of the real numbers, let $S(N)$ be the weak closure of the manifold spanned by $\{S(M): M$ $\subseteq N, M$ compact $\}$. Thus $S(N)$ is invariant under all bounded operators on $B$ commuting with $D_{A}$. In particular, $a \subseteq S(\{0\})$.

Lemma 3. Suppose $M$ is a real Borel set and $|\lambda|>1$ for all $\lambda \in M$. Then $S(M)=\{0\}$.

Proof. The proof reduces to the case when $M$ is compact. The hypothesis implies $M$ lies within the resolvent set for $D_{A}$. Thus for $\gamma \in M, D_{A}-\lambda I$ has a bounded inverse $B(\lambda)$ and the mapping $\lambda \rightarrow B(\lambda)$ is holomorphic on an open set containing $M$. This implies the existence of a constant $K$ such that $\|B(\lambda)\| \leqq K$ for all $\lambda \in M$. Choose $\epsilon$ with $0<\epsilon<K^{-1}$. For $\lambda \in M$ and $X \in S(\lambda, \epsilon),\|X\|=\left\|B(\lambda)^{n}\left(D_{A}-\lambda\right)^{n} X\right\|$ $\leqq(K \epsilon)^{n} K_{x}$ for all $n>0$ and thus $\|X\|=0$. Thus $S(M, \epsilon)=\{0\}$ and the same is true for $S(M)$.

Lemma 4. For Borel sets $M, N ; S(M) S(N) \subseteq S(M+N)$ and $S(M)^{*}$ $=S(-M)$.

Proof. Suppose $\epsilon>0$ and $X \in S(\lambda, \epsilon), Y \in S(\mu, \epsilon)$. Since $D_{A}$ is a derivation,

$$
\begin{aligned}
\left\|\left(D_{A}-(\lambda+\mu)\right)^{n} X Y\right\| & =\left\|\sum_{n}\left(C_{m}\left(\left(D_{A}-\lambda\right)^{m} X\right)\left(D_{A}-\mu\right)^{n-m} Y\right)\right\| \\
& \leqq \sum_{n} C_{m} \epsilon^{m} K_{X} \epsilon^{n-m} K_{Y}=(2 \epsilon)^{n} K_{X} K_{Y} .
\end{aligned}
$$

Thus $S(\lambda, \epsilon) S(\mu, \epsilon) \subseteq S(\lambda+\mu, 2 \epsilon)$.

Suppose now that $M, N$ are compact, $\lambda_{i} \in M, \mu_{j} \in N, X_{i} \in S\left(\lambda_{i}, \epsilon\right)$, $Y_{j} \in S\left(\mu_{j}, \epsilon\right)$, and $X=\sum X_{i}, Y=\sum Y_{j}$. Then $X Y=\sum \sum X_{i} Y_{j}$ so that $X Y \in S(M+N, 2 \epsilon)$. The sets of $X$ and $Y$ obtained in this way are weakly dense in $S(M, \epsilon)$ and $S(N, \epsilon)$, respectively. Since, in the weak operator topology, multiplication is continuous in each factor separately, we then have $S(M) S(N) \subseteq S(M, \epsilon) S(N, \epsilon) \subseteq S(M+N, 2 \epsilon)$. 
Since $\epsilon$ was arbitrary, this implies $S(M) S(N) \subseteq S(M+N)$ for compact $M$ and $N$. The general case follows immediately from this.

Induction on $n$ shows that $\left(\left(D_{A}-\lambda\right)^{n} X\right)^{*}=(-1)^{n}\left(\left(D_{A}+\lambda\right)^{n} X^{*}\right)$ for real $\lambda$ and $X \in ß$. Thus $S(\lambda, \epsilon)^{*}=S(-\lambda, \epsilon)$. A proof like that used above shows that $S(M)^{*}=S(-M)$.

Corollary. For each $\lambda>0, S([\lambda, 1])$ is a weakly closed nilpotent subalgebra of $B$. In fact, if $n$ is any integer with $n \lambda>1$, the product of any $n$ factors taken from $S([\lambda, 1])$ is zero.

Definitions. Let $S_{0}=U_{\lambda>0} S([\lambda, 1])$ and $\delta=S((0,1])$. Let $\Im$ be the algebraic sum of $a$ and $s$. By virtue of Lemma 4 the following assertions are evident:

(1) $J$ is a subalgebra of $B$.

(2) $S_{0}$ and $S$ are two-sided ideals of $J$.

(3) Every element of $S_{0}$ is nilpotent.

(4) $S$ is the weak closure of $S_{0}$.

It will be shown below that $J$ is a maximal hyperreducible triangular algebra with diagonal $a$.

Let $E$ be the real spectral measure associated with $A$ and let $E_{\lambda}=E((-\infty, \lambda))$. Then $E_{\lambda}=\sup E_{\mu}$ where $\mu<\lambda$ so that $E_{0}=0$. Also $E_{\lambda}=1$ for $\lambda>1$ and the set of $E_{\lambda}$ generates $Q$ as a von Neumann algebra.

For $\lambda$ real and $\epsilon>0$ let $F(\lambda, \epsilon)$ be the set of vectors $u \in \mathcal{F}$ such that $\left\|(A-\lambda)^{n} u\right\| \leqq \epsilon^{n} K_{u}$ for some constant $K_{u}$ and $n=1,2, \cdots$. If we let $\mathscr{F}(N, \epsilon)$ be the closed subspace spanned by $\{\mathscr{F}(\lambda, \epsilon): \lambda \in N\}$ then it follows from a result in [3, pp. 66-69] that if $N$ is compact, the range of $E(N)$ equals $\mathcal{F}(N)=\bigcap_{\epsilon>0} \mathcal{F}(N, \boldsymbol{\epsilon})$. For a Borel set $N$ we use $\mathcal{E}(N)$ to denote the range of the projection $E(N)$.

Theorem 2. For Borel sets $M$ and $N, S(M) \mathcal{E}(N) \subseteq \mathcal{E}(M+N)$.

Proof. Suppose $\lambda, \mu$ are real and $\epsilon>0$. Choose $u \in F(\mu, \epsilon)$ and $X \in S(\lambda, \epsilon)$. Then

$$
\begin{aligned}
\left\|(A-(\lambda+\mu))^{n} X u\right\| & =\left\|\left(\left(D_{A}-\lambda\right)+\left(R_{A}-\mu\right)\right)^{n} X u\right\| \\
& =\left\|\sum_{n} C_{m}\left(D_{A}-\lambda\right)^{n-m} X(A-\mu)^{m} u\right\| \\
& \leqq \sum_{n} C_{m} \epsilon^{m} K_{X} \epsilon^{n-m} K_{u}=(2 \epsilon)^{n} K_{X} K_{u} .
\end{aligned}
$$

Thus $S(\lambda, \epsilon) \mathscr{F}(\mu, \epsilon) \subseteq \mathscr{F}(\lambda+\mu, 2 \epsilon)$.

An argument like that used in the proof of Lemma 4 can now be used to prove the assertion of the theorem when $M$ and $N$ are com- 
pact. By using approximations with compact subsets the general assertion follows from this.

CoRollary. For any real $\lambda$ the range of $1-E_{\lambda}$ is invariant under $J$.

Lemma 5. (1) Suppose $0 \leqq \mu<\lambda \leqq 1$. Then $\left(1-E_{\lambda}\right) \otimes E_{\mu} \subseteq S_{0}$.

(2) For any $\lambda,\left(1-E_{\lambda}\right) \beta E_{\lambda} \subseteq \delta$.

Proof. (1) Suppose $\epsilon>0$. Let $\lambda_{0}=\lambda$ and choose $\lambda_{1}, \cdots, \lambda_{n}$ with $\lambda_{n}>1$ such that each of the intervals $\left[\lambda_{0}, \lambda_{1}\right), \cdots,\left[\lambda_{n-1}, \lambda_{n}\right)$ has length less than $\epsilon$. Let $E_{i}=E\left(\left[\lambda_{i-1}, \lambda_{i}\right)\right), i=1, \cdots, n$. Then $1-E_{\lambda}$ $=\sum E_{i}$. Similarly we can partition $[0, \mu)$ into $m$ disjoint intervals of length less than $\epsilon$ and express $E_{\mu}$ as the sum of $m$ mutually orthogonal projections $F_{j} \in Q$. Choose arbitrary points $\alpha_{i}, \beta_{j}$ in the $i$ th and $j$ th intervals of the partitions of $\left[\lambda_{0}, \lambda_{n}\right)$ and $[0, \mu)$, respectively. Then $\alpha_{i}-\beta_{j} \geqq \lambda-\mu>0$.

For $X \in \Re,\left(1-E_{\lambda}\right) X E_{\mu}=\sum \sum E_{i} X F_{j}$. For a positive integer $k$,

$$
\begin{aligned}
\left\|\left(D_{A}-\left(\alpha_{i}-\beta_{j}\right)\right)^{k} E_{i} X F_{j}\right\| & =\left\|\left(\left(L_{A}-\alpha_{i}\right)-\left(R_{A}-\beta_{j}\right)\right)^{k} E_{i} X F_{j}\right\| \\
& \leqq \sum_{k} C_{p}\left\|\left(A-\alpha_{i}\right)^{p} E_{i}\right\|\|X\|\left\|\left(A-\beta_{j}\right)^{k-p} F_{j}\right\| \\
& \leqq(2 \epsilon)^{k}\|X\| .
\end{aligned}
$$

Thus $E_{i} X F_{j} \in S\left(\alpha_{i}-\beta_{j}, 2 \epsilon\right) \subseteq S([\lambda-\mu, 1]) \subseteq S_{0}$.

(2) For any $X$ and $0 \leqq \lambda \leqq 1,\left(1-E_{\lambda}\right) X E_{\lambda}$ is a weak limit of operators of the form $\left(1-E_{\lambda}\right) X E_{\mu}$ where $\mu<\lambda$, hence lies in $S$.

THEOREM 3. I is a maximal hyperreducible triangular algebra with diagonal a. Moreover, every algebra of this type (acting on a separable space) is obtained by a construction like that given above.

Proof. Let $\Im_{1}$ be the set of all $X \in B$ which leave the range of $1-E_{\lambda}$ invariant for all $\lambda$. It is shown in [5, Theorem 3.1.1] that $J_{1}$ is a maximal hyperreducible triangular algebra and we have proved that

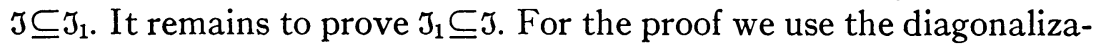
tion process developed by von Neumann and generalized by Kadison and Singer in [4, pp. 386-387].

Let $\left\{\lambda_{n}\right\}$ be a countable dense subset of $[0,1]$ and $E_{n}=E_{\lambda_{n}}$. Then $\left\{E_{n}\right\} \cup\{1\}$ generates $a$ as a von Neumann algebra. Define the projection $P_{n}$ on $B$ by $P_{n}(X)=E_{n} X E_{n}+\left(1-E_{n}\right) X\left(1-E_{n}\right)$. Then $\left\|P_{n}(X)\right\| \leqq\|X\|$ and $P_{n}$ leaves $\Im_{1}$ invariant. For $X \in \Im_{1}, X=P_{n}(X)$ $+\left(1-E_{n}\right) X E_{n}$ and the latter term is in $\mathcal{S}$ by virtue of Lemma 5 . Let $X_{0}=X$ and $X_{n}=P_{n}\left(X_{n-1}\right)$ for $n \geqq 1$. Then $X_{n} \in J_{1}$ and, by induction on $n$, we have $X=X_{n}+S_{n}$ where $S_{n} \in \mathcal{S}$. Because of the compactness of the unit sphere of $B$ in the weak topology a subsequence $\left\{X_{n_{k}}\right\}$ 
converges weakly to an element $B \in B$ and thus $\left\{S_{n_{k}}\right\}$ converges weakly to some $S \in S$. Since $B$ will commute with all $E_{n}, B \in Q$ and hence $X=B+S$ lies in $J$ so that $J_{1} \subseteq J$.

If $\zeta$ is any maximal hyperreducible triangular algebra with diagonal a, a modification of the proof of Theorem 3.3.1 in [5] shows that it is possible to construct a spectral family $\left\{E_{\lambda}: 0 \leqq \lambda \leqq 1\right\}$ of projections in $Q$ such that $J$ is the set of all $X \in B$ with $E_{\lambda} X=E_{\lambda} X E_{\lambda}$ for all $\lambda$. If we define $A$ by $A=\int \lambda d E_{\lambda}$, then $Q=\left\{X: D_{A} X=0\right\}$, and if we use this $A$ in the construction, the result will be $\mathfrak{J}$, as shown in the preceding part of the proof.

\section{Determination of $a \cap s$.}

REMARK. In connection with the structure of $J$ the question arises as to necessary conditions in order that the sum $a+\delta$ be direct. A complete solution is given here for the case when $B$ is of Type I or II. A question which seems to be related to this arises regarding the possibility of expressing each $X \in J$ as $X=B+S$ where $B \in Q$ and $S$ is quasi-nilpotent. The answer to this is not known to us and, because of the nonadditivity of quasi-nilpotence, it is conceivable that it can be accomplished even when $a \subseteq S$.

Lemma 6. Suppose $B$ is the algebra of all bounded operators on $\mathrm{TC}$ and the point spectrum of $A$ is empty. Then $a \subseteq S$.

Proof. Let $\mathscr{g}=a \cap S$. Then $\mathscr{g}$ is an ideal of $Q$ and thus it is sufficient to prove that $1 \in \delta$. Because of [5, Theorem 3.3.1] we may assume that $Q$ is the algebra of all bounded measurable functions in $L^{2}([0,1])$ and $E_{\lambda}$ is multiplication by the characteristic function of the interval $[0, \lambda)$.

Let $\varepsilon$ be the set of all operators on $L^{2}$ which are finite sums $\sum f_{i} \otimes g_{i}$ where $f_{i}, g_{i} \in L^{2}$ and $\left(f_{i} \otimes g_{i}\right) h=\left(h, g_{i}\right) f_{i}$. By making use of the canonical trace on $B, \mathcal{E}$ can be identified with the set of all weakly continuous linear functionals on $B[1$, p. 388]. Let $\beta=\{T: T \in \mathcal{E}, \operatorname{Tr}(T X)=0$ for all $X \in \mathcal{S}\}$. Since $\mathcal{S}$ is weakly closed, $\mathcal{S}=\{X: \operatorname{Tr}(T X)=0$ for all $T \in \mathcal{P}\}$. Thus $1 \in \mathcal{S}$ if and only if $\operatorname{Tr}(T)=0$ for all $T \in \mathcal{}$.

Suppose $T \in \mathcal{}$. For $f, g \in L^{2}$, Lemma 5 implies $\left(1-E_{\lambda}\right) f \otimes g E_{\lambda} \in \mathcal{S}$ so that $\operatorname{Tr}\left(T\left(1-E_{\lambda}\right) f \otimes g E_{\lambda}\right)=0$ and this implies $\left(E_{\lambda} T\left(1-E_{\lambda}\right) f, g\right)$ $=0$. Since $f, g$ were arbitrary this shows that $E_{\lambda} T\left(1-E_{\lambda}\right)=0$ for any $\lambda$ and thus $T \in J$. Since $T$ is of trace class there is a function $K$ in $L^{2}$ of the unit square such that $T f(x)=\int K(x, y) f(y) d y$ for $f \in L^{2}$. Since $T \in J$ it is easy to see that $K$ is of Volterra type, i.e., $K(x, y)=0$ for $y>x$. A well-known theorem of integral equations [6, pp. 10-11] implies $T$ is quasi-nilpotent and, since the range of $T$ is finite-dimensional, this means $T$ is nilpotent so that $\operatorname{Tr}(T)=0$. 
TheOREM 4. (1) If $B$ is a factor of Type II, $Q \cap S=\{0\}$.

(2) Suppose $B$ is of Type $\mathrm{I}_{\infty}$. Let $M$ be the set of characteristic values of $A$ and $E=E([0,1]-M)$. Then $a \cap S=E Q$. Thus $a \cap S=\{0\}$ if and only if $A$ has pure point spectrum.

Proof. (1) We consider only the case when $B$ is finite. The infinite case can be deduced from this or obtained by a refinement of the argument given here. For $X, Y \in B$ let $(X, Y)=\operatorname{Tr}\left(X Y^{*}\right)$ where $\operatorname{Tr}$ is the canonical trace on $B$. Then $B$ becomes a pre-Hilbert space. Since $a \subseteq S(\{0\})$ the assertion follows from a more general result: If $M$ and $N$ are disjoint Borel sets then $(S(M), S(N))=0$. By using approximations with compact subsets we may assume both $M$ and $N$ are compact. Then $M-N$ is a compact set bounded away from zero and $S(M) S(N)^{*} \subseteq S(M-N)$. Since $S(M-N)$ is spanned by nilpotent elements of $B$ it is sufficient to prove that any nilpotent $X \in B$ has trace zero. However, this is a consequence of a result proved in [2, p. 108] where it is shown that $\operatorname{Tr}(X)$ belongs to the convex hull of the spectrum of $X$.

(2) Let $\mathfrak{g}=\boldsymbol{S} \cap \mathfrak{Q}$. Then $\mathscr{g}$ is a weakly closed ideal of $\mathfrak{Q}$. Suppose $\mu$ is a characteristic value for $A$ and $P=E(\{\mu\})$. Then $P$ is a minimal projection in $Q$ and thus either $P \in g$ or $P \mathscr{g}=0$. Choose $\lambda>0$ and $S \in S([\lambda, 1])$. Then Theorem 2 implies $S P(\mathfrak{H C}) \subseteq E(\{\mu\}+[\lambda, 1])(\mathfrak{F C})$ so that $P v=v$ implies $(S v, P v)=0$. But then $\|(P-S) v\|^{2}=\|v\|^{2}+\|S v\|^{2}$ $\geqq\|v\|^{2}$. Since $\lambda$ was arbitrary this shows that $P$ is not contained in the strong closure of $S_{0}$. However, the result of [1, Note 1] shows that the strong and weak closures coincide and thus $P \notin S$. Then $P g=0$ and it follows that $\mathfrak{g} \subseteq E$ Q. Let $J^{\prime}=E J E$. Then $J^{\prime}$ is a maximal hyperreducible triangular algebra of operators with diagonal $E Q$ on the Hilbert space $E \mathcal{H C}$. Since $E A$ has no point spectrum on $E \mathcal{H}$, Lemma 6 implies $E Q \subseteq E S E$ and hence $E Q \subseteq g$.

\section{REFERENCES}

1. J. Dixmier, Les fonctionnelles linéaires sur l'ensemble des opérateurs bornés d'un espace de Hilbert, Ann. of Math. (2) 51 (1950), 387-408.

2. - Les algèbres d'opérateurs dans l'espace Hilbertien, Gauthier-Villars, Paris, 1957.

3. P. Halmos, Introduction to Hilbert space, Chelsea, New York, 1951.

4. R. Kadison and I. Singer, Extensions of pure states, Amer. J. Math. 81 (1959), $383-400$.

5. - Triangular operator algebras, Amer. J. Math. 82 (1960), 227-259.

6. F. Tricomi, Integral equations, Interscience, New York, 1957.

Macalester College 